The Egyptian Journal of Hospital Medicine (October 2019) Vol. 77 (1), Page 4688-4691

\title{
Preoperative Assessment of Crystalline Lens by A-Scan Quantitative Echography and Its Correlation with Phacoemulsification Parameters
}

\author{
Doaa A. Mahmoud, Hanan S. Hegazy
}

Department of Ophthalmology, Faculty of Medicine for Girls, Al-Azhar University, Cairo, Corresponding author: Doaa A. Mahmoud, Tel: +20 122610 0310; e-mail: doaamahmoud75@ gmail.com

\begin{abstract}
Purpose: To evaluate the correlations between the total ultrasound energy consumed during phacoemulsification with various preoperative parameters, including the best- corrected distant visual acuity (BCDVA) and quantified metrics taken from A-scan ultrasound biometry. Patients and methods: A prospective observational interventional study included 40 eyes of 40 consecutive patients scheduled for cataract surgery. We assessed the lens internal reflectivity in the A-scan echograms within a range of $0 \%$ to $100 \%$, and calculated the mean of all spikes. All eyes with cataract underwent clear corneal phacoemulsification. At the end of the operation we recorded the phacoemulsification time and mean percent power. The correlations of echographic and phacoemulsification data were assessed. Results: Quantitative echography in the 40 studied eyes with different types of cataract revealed acoustic reflections having various configurations according to the biomicroscopic type of cataract, i.e. cortical, nuclear and posterior subcapsular cataract. The mean spikes were $31.027 \% \pm 25.5908$, the mean lens thickness was $4.291 \pm 0.6166$, the mean best corrected distant visual acuity in decimal was $0.205 \pm 0.1435$, the mean phaco power\% was $6.400 \pm 3.0701$ and the mean phaco time is 84.225. There was statistically insignificant correlation between spikes\% and phaco power\% in the study group (p>0.05). There was statistically insignificant correlation between mean spikes $\%$ and lens thickness in the study group ( $>0.05)$.There was statistically insignificant correlation between spikes $\%$ and phaco time (in seconds) in the study group $(\mathrm{p}>0.05)$. Conclusion: With regards to the predictive value of lens hardness changes in the acoustic properties identified by our method, it is not correlated with the ultrasound energy required intraoperatively. The A-scan is helpful for the clinical prediction of lens opacity location, but not predictive of lens hardness quantification.
\end{abstract}

Keywords: crystalline lens, A-scan quantitative echography, phacoemulsification.

\section{INTRODUCTION}

Cataract is clouding of the crystalline lens that reduces the amount of incoming light and impairs visual perception. Phacoemulsification is the most common surgical method for treating advanced cataracts. The optimal phacoemulsification energy is determined by the lens hardness ${ }^{(1)}$. A detailed evaluation of cataract density is essential in surgery planning in order to improve visual outcomes and to avoid possible intraoperative and postoperative complications ${ }^{(2)}$. In the past quantification of crystalline lens hardness before cataract removal has been attempted by several researchers. These have been in humans and in animals, and have used a variety of imaging modalities ${ }^{(3)}$. Ultrasound is an effective approach to an objective evaluation of the hardness of the cataractous lenses. The frequently applied methods are based on measuring ultrasonic attenuation and sound speed, which tend to increase with the increased hardness of the cataract lens ${ }^{(4)}$. It is already known that A-scan examination of cataract lens will show one or more spikes originating from the lens, appearing between the highly reflective anterior and posterior lens spikes ${ }^{(3)}$. Brazitikos et al. ${ }^{(5)}$ have documented the importance of A-scan lens echography in classifying the distinct category of white senile cataracts and in preoperatively evaluating the potential risks of performing phacoemulsification in these eyes.

\section{PATIENTS AND METHODS}

A prospective observational interventional study included 40 eyes of 40 consecutive patients scheduled for cataract surgery. It was carried out at Al-Azhar university hospital between (March 2018 to September 2018). Ethical approval: The study was approved by the Ethics Board of Al-Azhar University and an informed written consent was taken from each participant in the study.

\section{Inclusion criteria:}

- Clinical diagnosis of cataract by slit lamp.

- Mental and physical capacity to undergo the test.

- Normal posterior segment. Exclusion criteria:

- Previous eye surgery.

- Coexistence of ocular pathology other than cataract (eg. Vitreous hemorrhage, retinal detachment, and elevated choroidal lesion ).

The study protocol adhered to the tenets of the declaration of Helsinki and was approved by the ethical board of Al-Azhar University. Informed written consent was taken from each participant in the study.

Each subject was undergone:

I. Full ophthalmic examination including : Visual acuity using Snellen visual acuity chart which was converted to decimal notation for statistical analysis, 
slit lamp examination for cataract grading using LOCSII as described by Chylack et al. ${ }^{(6)}$,

intraocular pressure measurement using Goldmann applanation tonometry and dilated fundus examination if possible.

\section{II.}

A-scan echography of the lens:

The acoustic structure of lenses was recorded during the preoperative axial length measurement, quantitative A-scan echography using a contact applanation method (Mentor ${ }^{\circledR}$ AdventTM A/B System 10-MHz biometry probe), with the probe positioned at the center of the cornea, for the sound beam to be directed perpendicular to the lens.

We obtained in all echograms near to $100 \%$ spikes (compared to the anterior-corneal and posterior retinal spikes) from the anterior and posterior lens surface and the height of all internal acoustic spikes of the lens was measured. The calculation of the internal spikes was made within a range of $0 \%$ to $100 \%$ with the vitreous, the parameter employed were mean height of internal lens spikes and the height of (L1) spike, the ratio of these parameters were the main index of interest (mean of spikes /L1ratio) Figure (1).

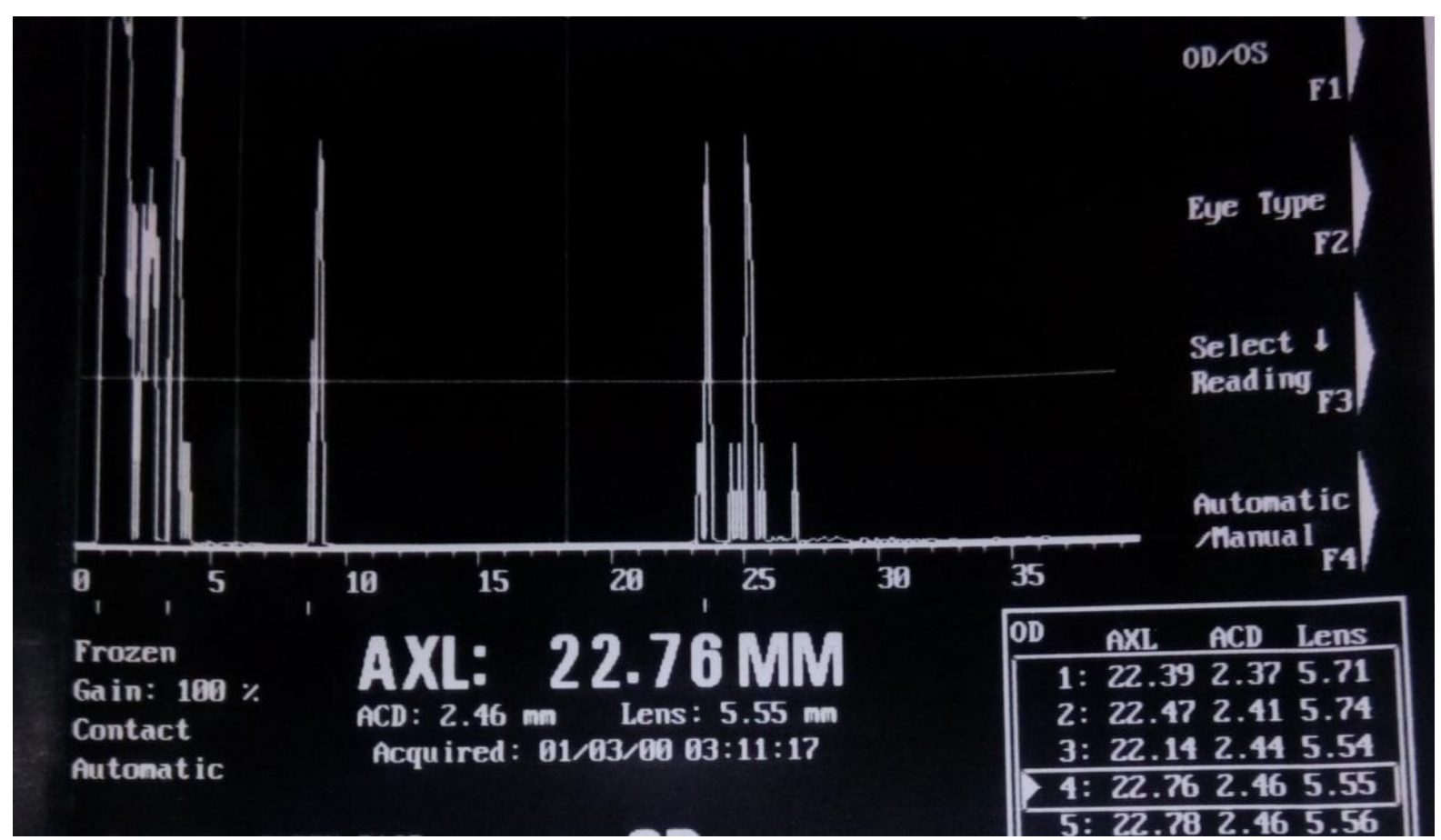

Figure (1): Ultrasound A-scan echogram of corticonuclear cataract showing multiple internal spikes. The lens thickness was $5.5 \mathrm{~mm}$ there are 3 high internal spikes $(2.6,0.5,1.4)$ the mean of the internal lens spike is $124.8(41.6 \%)$, mean phaco power $4 \%$ and phaco time 2 seconds.

Phacoemulsification was performed in all cases by the same surgeon under local anesthesia with a peribulbar injection of a mixture of lidocaine $1 \%$ and bupivacaine $0.5 \%$. A superior temporal, near limbus, three- step, $2 \mathrm{~mm}$ long corneal tunnel approach was employed for surgery. Following capsulorhexis and cortical cleaving hydrodissection, phacoemulsification of the nucleus using the linear mode was performed through an incision of $2.75 \mathrm{~mm}$ with (Whitestar Signature ${ }^{\circledR}$ Pro phacoemulsification system) using a 30 degrees ABS tip. The maximum phaco power was arbitrarily set to $60 \%$ in all cases.

Stop and chop was the standard phacoemulsification technique in all patients. Phacoemulsification of the nucleus was followed by cortex removal. Complicated cases by posterior capsule rupture and vitreous loss were excluded. At the end of the operation the phacoemulsification time (seconds) and mean percent power was recorded (they were displayed at the LCD panel of the system).

\section{Statistical analysis}

To assess the relation between echographic, optical and phacoemulsification variables, analysis of data was performed using SPSS v. 22 (Statistical Package for Scientific Studies) for Windows \& MedCalc v. 18.

- The significance of the results was assessed in the form of P-value that was differentiated into:

- Non-significant when P-value >0.05.

- $\quad$ Significant when P-value $\leq 0.05$. 
- Highly significant when P-value $\leq 0.01$.

\section{RESULTS}

The study included 40 eyes of 40 patients(18 male and 22 female). All of them underwent phacoemulsification cataract extraction and in-the-bag implantation of the foldable IOL.

The mean age of males was $60.611 \pm 9.369$ years range (33-75) and the mean age of female was $56.318 \pm 7.74$ years range (37-66). The mean spikes were $31.027 \% \pm 25.5908$, the mean lens thickness was 4.291 \pm 0.6166 , the mean best -corrected distant visual acuity in decimal was $0.205 \pm 0.1435$, the mean phaco power\% was $6.400 \pm 3.0701$ and the mean phaco time is 84.225 (Table 1).

Table (1): Mean and SD of Preoperative surgical parameters.

\begin{tabular}{|c|l|c|}
\hline Parameter & Mean & SD \\
\hline BCDVA_decimal & 0.205 & 0.1435 \\
\hline Mean Spikes \% & 31.027 & 25.5908 \\
\hline Lens thickness & 4.291 & 0.6166 \\
\hline Phaco power \% & 6.400 & 3.0701 \\
\hline Phaco time (seconds) & 84.225 & 79.0402 \\
\hline
\end{tabular}

There was a statistically insignificant correlation between BCDVA and other parameters (Table 2).

Table (2): Correlation between BCDV and other parameters

\begin{tabular}{|l|c|c|}
\hline \multirow{2}{*}{ Parameter } & \multicolumn{2}{|c|}{ BCDVA (decimal) } \\
\cline { 2 - 3 } & Correlation (r) & P.value \\
\hline Spikes \% & 0.03592 & 0.8258 \\
\hline Lens thickness & 0.05148 & 0.7524 \\
\hline Phaco power \% & 0.04108 & 0.8013 \\
\hline Phaco time (seconds) & -0.2580 & 0.1079 \\
\hline
\end{tabular}

There was a statistically insignificant correlation between lens thickness and other parameters (phaco power, phaco time) in the study group (P.>0.05). There were statistically insignificant correlations between spikes\%, lens thickness, phaco power\% and phaco time (Table 3) Figure (2).

Table (3) : Correlations between spikes \%, Lens thickness, Phaco power $\%$ and Phaco time

\begin{tabular}{|c|c|c|}
\hline \multirow{2}{*}{ Parameter } & \multicolumn{2}{|c|}{ Spikes \% } \\
\cline { 2 - 3 } & Correlation (r) & P.value \\
\hline Lens thickness & -0.1882 & 0.245 \\
& & \\
\hline Phaco power \% & 0.1144 & 0.4822 \\
\hline Phaco time (seconds) & 0.1955 & 0.2268 \\
\hline
\end{tabular}
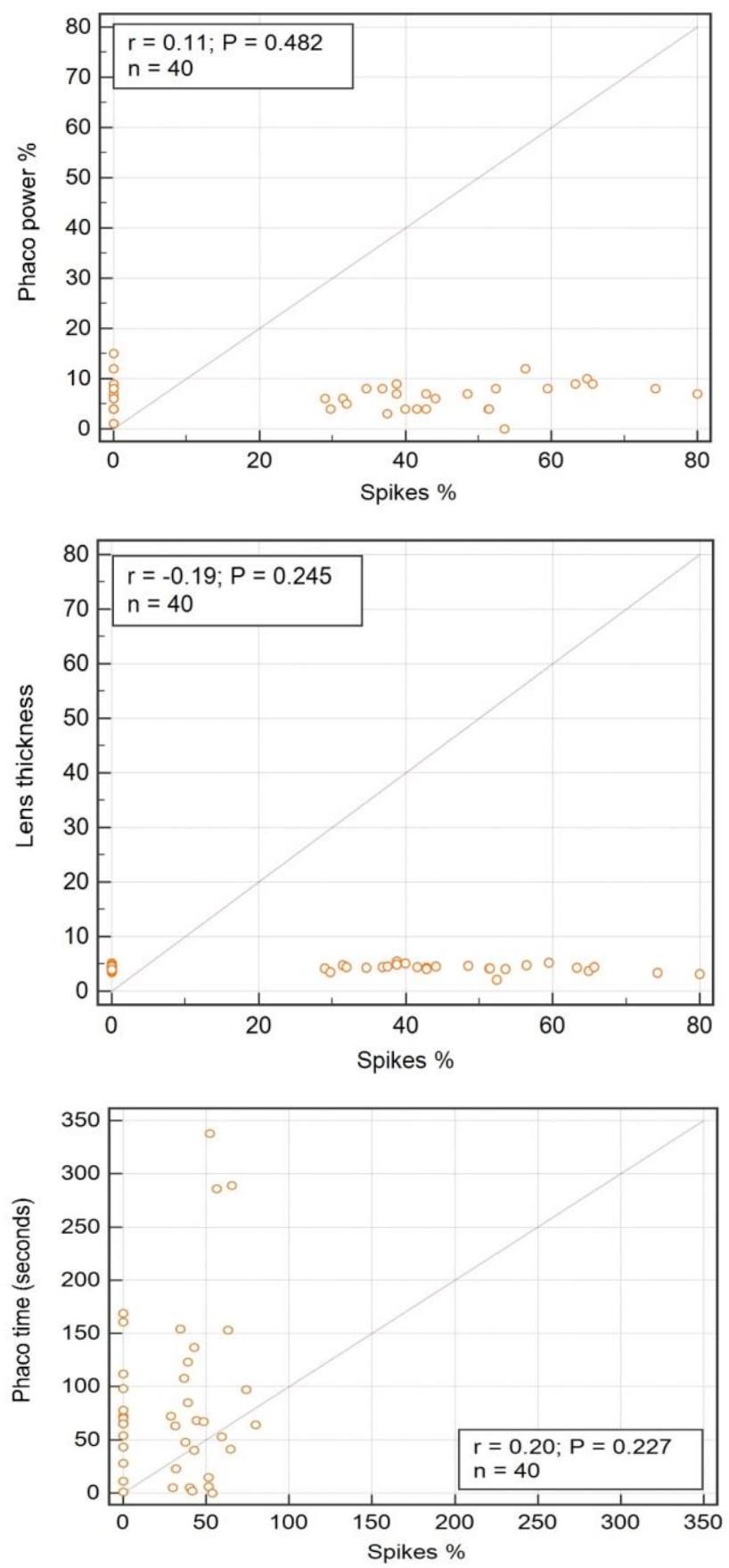

Figure (2): Pearson correlation between spikes, lens thickness, Phaco power \% and Phaco time.

\section{DISCUSSION}

Ultrasound is an effective approach to objective evaluation of the hardness of the cataractous lenses ${ }^{(7)}$. In general, the echo intensity of the cataract lens differs from that of the normal lens, for optical opacities produce acoustic inhomogeneity, with the cataract area exhibiting high echogenicity ${ }^{(1)}$. The frequently applied methods are based on measuring ultrasonic attenuation 
and sound speed, which tend to increase with the hardness of the cataractous lens ${ }^{(8)}$.

In our study, we found that there was a statistically insignificant correlation between BCDVA (decimal and log MAR), mean spike, lens thickness, phaco time, mean phaco power $\%$ and cataract grading. This was in disagreement with what stated by Tsaousis et $\boldsymbol{a l} .{ }^{(9)}$, who found that there was a significant correlation between the phaco time, mean phaco power and the BCDVA. Although it is reasonable to assume that visual acuity is affected by cataract changes proportionally to alterations in the optical properties of the lens with increased scattering. This appears to be more important than the changes in the acoustic behaviour of the cataractous lens. This findings could reflect that the optical properities of the lens appears to be more important than its acoustic behavior .

With regards to the predictive value of lens hardness, lens thickness and mean spikes detected by the ultrrasound did not correlate with the ultrasound energy required intraoperatively, unlike Brazitikos $\boldsymbol{e t}$ al. ${ }^{(10)}$, who showed that the simple non-invasive method of A-scan echography may be adjunctive to the biomicroscopic examination in the preoperative evaluation of cataracts.

As regard lens thickness, it is not a measure for sound attenuation or sound propagation through lens and it is affected by patient accommodation, so it can't be a reflection to lens hardness.

The relationship between lens clarity and hardness is uncertain. Indeed they may be independent of each other. Alterations in the acoustic properties of lenses may occur by mechanisms which do not affect the hardness of the lens ${ }^{(9)}$. So, lens hardness is still challenging despite many studies both in vitro and in vivo. Methods for in vivo evaluation of lens hardness and its relationship with the clincal appearance, ultrasonic attenuation and optical properties must be further developed.

\section{CONCLUSION}

With regards to the predictive value of lens hardness changes in the acoustic properties identified by our method, it is not correlated with the ultrasound energy required intraoperatively. The A-scan is clinically predicting opacified lens, but it is not able to provide information regarding lens hardness quantification.

\section{ACKNOWLEDGEMENT}

Aspecial thanks to Prof. Dr. Fatma A. Atwa

(Department of Ophthalmology, Faculty of medicine for girls, Al-Azhar University) for her great support and help during the whole work.

\section{REFERENCES}

1. Coleman DJ, Silverman RH, Lizzi FL et al. (2006): Ultrasonography of the eye and orbit. Philadelphia, PA: Lippincott Williams \& Wilkins: pp.210-212.

2. Chylack LT Jr, Wolfe JK, Singer DM et al. (1993): The lens opacities classification system III. The longitudinal study of cataract study group. Arch Ophthalmol., 111: 831-836.

3. Wong WL, Li X, Li J, Cheng CY et al. (2013): Cataract conversion assessment using lens opacity classification system III and Wisconsin cataract grading system. Invest Ophthalmol Vis Sci., 54(1): 280-287.

4. Huang CC, Ameri H, DeBoer C et al. (2007): Evaluation of lens hardness in cataract surgery using high frequency ultrasonic parameters in vitro. Ultrasound Med Biol., 33:1609-1616.

5. Brazitikos PD, Tsinopoulos IT, Papadopoulos NT et al (1999): Ultrasonographic classification and phacoemulsification of white, senile cataracts. Ophthalmology, 106:2178-2183.

6. Chylack LT Jr, Leske MC, McCarthy D et al. (1989): Lens opacities classification system II (LOCS II). Arch Ophthalmol., 107(7): 991-7.

7. Tabandeh H, Wilkins M, Thompson G et al. (2000): Hardness and ultrasonic characteristics of human crystalline lens. J Cataract Refract Surg., 26:838-41.

8. Huang C, Zhou Q, Ameri H et al. (2007): Determining the acoustic properties of the lens using a high-frequency ultrasonic needle transducer. Ultrasound Med Biol., 33(12):1971-1977.

9. Tsaousis KT, LamporgiannisLP, Dimitrakos SA et al. (2016): Preoperative evaluation of human crystalline lens hardness using A-scan ultrasound biometry: Apilot study. Int J Ophthalmol., 9(10):1521-1523

10. Brazitikos PD, Androudi S, Papadopoulos NT et al. (2003): A-scan quantitative echography of senile cataracts and correlation with phacoemulsification parameters. Current Eye Research, 27(3): 175-181. 